\title{
Coupling Empowerment Based Application of Extension Method for Geothermal Potential Assessment
}

\author{
Qing Zhang $\mathbb{D}^{1},{ }^{1}$ Weiya Ge, ${ }^{1}$ Fujin Tian, ${ }^{1}$ Yunfeng Li, ${ }^{1}$ Lili Hou, ${ }^{1}$ \\ Lujuan $\mathrm{Li}^{2}$ and Jianming $\mathrm{Li}^{3}$ \\ ${ }^{1}$ Nanjing Center, China Geological Survey, Nanjing 210016, China \\ ${ }^{2}$ Institute of Karst Geology, CAGS, Guilin 541000, China \\ ${ }^{3}$ College of Construction Engineering, Jilin University, Changchun 130026, China \\ Correspondence should be addressed to Qing Zhang; sys8633@126.com
}

Received 10 September 2017; Revised 7 January 2018; Accepted 15 January 2018; Published 15 February 2018

Academic Editor: Amirhossein Mosaffa

Copyright (C) 2018 Qing Zhang et al. This is an open access article distributed under the Creative Commons Attribution License, which permits unrestricted use, distribution, and reproduction in any medium, provided the original work is properly cited.

Plenty of mathematics researches provide feasibility to calculate the weights of geothermal controlling factors and have been applied in geothermal potential assessment. In order to avoid the disadvantages of subjective and objective weighting calculation methods, an extension theory integrated weighting method was put forward, by combining with the process of AHP and mean variance method. The improved method can reach an agreement on subjective understanding of impact factors' roles and data-based calculation weights. Then by replacing the point values with intervals, the extension theory was used in classification of geothermal assessment, according to extension judgment matrix. The evaluation results showed perfect performance in classification of impact factors, especially in Wudalianchi area, where 10 out of 11 selected impact factors agreed well with the actual evaluation grades. The study can provide a guidance for primary stage of geothermal investigation including the impact factor selection, weights calculation for impact factors, and the factors' classification in geothermal assessment.

\section{Introduction}

Due to fossil fuel energy such as oil, coal, and natural gas which may account for $>85 \%$ of entire energy consumption, the issues of energy and environment still need to be given deep consideration even if this adverse trend has been alleviated for now $[1,2]$. In China, a great deal of geothermal energy remains undiscovered or fully exploited, thus fossil fuel energy can be deemed to be a competitive one undoubtedly $[3,4]$. Since the 1990s, geothermal resources have been widely applied in various fields, improved people's lives significantly, and promoted social development drastically [58]. According to a report proposed by the Chinese Academy of Engineering (2013), the entire utilized shallow geothermal energy $\left(5.0 \times 10^{3} \mathrm{mwt}\right)$ with medium and low temperatures is expected to increase threefold by 2050 . In addition, the installed capacity for generating high temperature geothermal energy especially for enhanced geothermal system (EGS) has been greatly increased significantly in the last few decades. Thus, considering the important role of geothermal resources, the processing treatments including the geothermal identification, impact factor selection, geothermal potential regions assessment, and utilization conditions evaluation should be performed in early stage exploration [9].

In general, the commonly applied methods for geothermal potential evaluation usually are fuzzy comprehensive evaluation method [10], Monticello method [11], the back propagation (BP) neural network [12], the weights of evidence (W of E) method [13], logistic regression model, and so forth [14] (Williams et al., 2008). In these methods, the impact factors are single fixed and some selected databases are not quantitative. In addition, some methods are not easy to implement because the used impact factors not only have different influencing roles of the surrounding conditions but also possess inner nonlinear relationship between the factor pairs [15-17]. Thus, the impact factors' corresponding weights should be taken into account before modeling application. The assignment of weights which can be obtained in these 


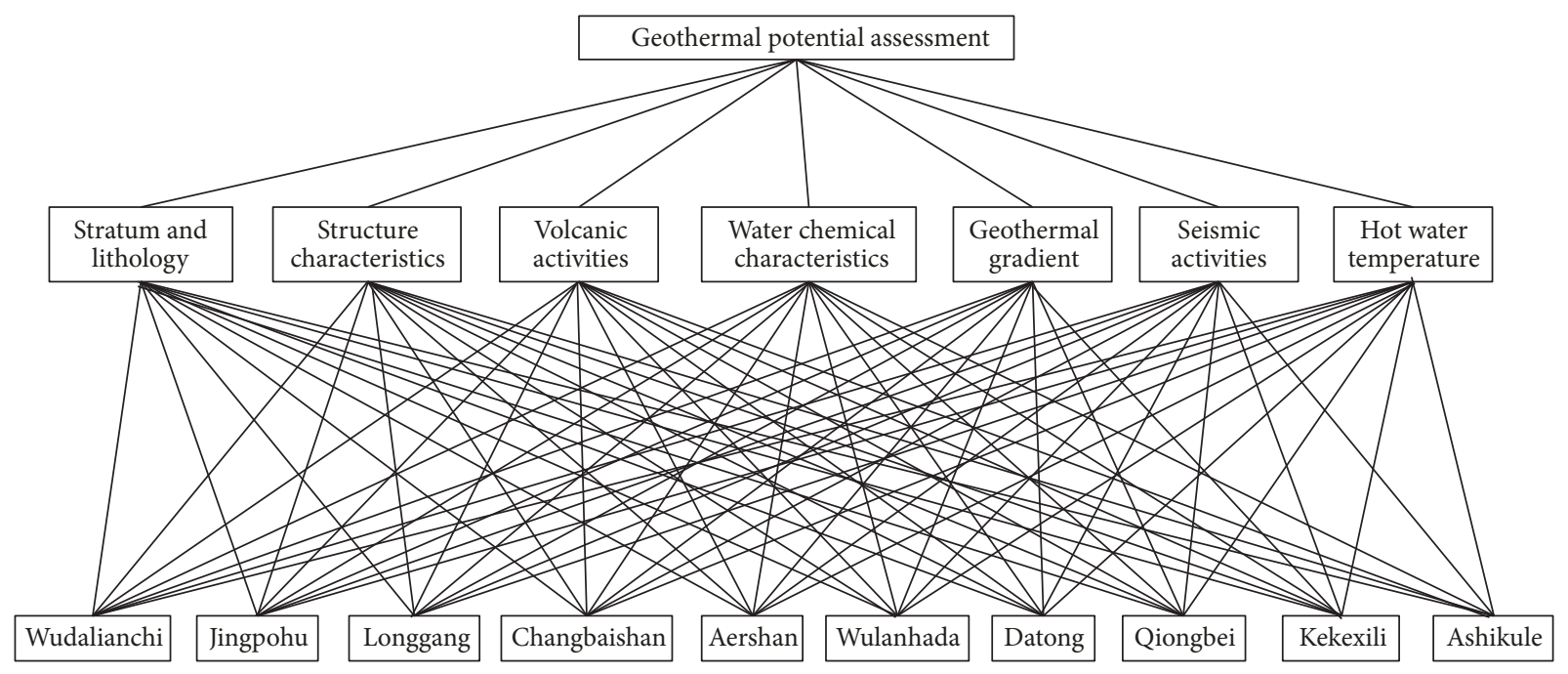

FIGURE 1: Hierarchical structure model of geothermal potential.

methods usually embraces two cases: judging on the basis of the subjective experience or calculating coefficients directly according to the objective database of factors. Both the two cases resulted in an unreliability of weight for each impact factor because of the artificial uncertainty or data deficiency $[18,19]$. Therefore, a coupling empowerment extension theory was proposed as follows where the limitations on statistical methods or knowledge driven methods can be avoided [20]. By making full use of the subjective selection ability in the analytic hierarchy process (AHP) and the objective ability of digging out source data in the mean variance method, the obtained index weights for impact factors were calculated, reflecting both subjectivity and objectivity. Then, the singular data were also removed reasonably in data processing. Finally, the extension method combining the weights was put forward in assessment of the geothermal potential regions.

\section{Methodology}

2.1. Analytic Hierarchy Process. AHP was first proposed by Saaty [21], and the system methods have been successfully applied to make optimization decisions by decomposing complex problems into several multiple indicator levels [22, 23]. Thus, the AHP method can also be used here in decision system to determine the weights of impact factors with characteristics of various objective distributions, multicriteria evaluation, and a situation that is difficult to quantify. Undoubtedly, the method was applied in a straightforward manner and performed easily. A schematic diagram of AHP for geothermal potential assessment was shown in Figure 1, where each subjective weight for impact factors was calculated by the analytic hierarchy process. The selected 11 representative study areas located in China, respectively, were Wudalianchi, Jingpohu, Longgang, Changbaishan, Aershan, Wulanhada, Datong, Qiongbei, Kekexili, Ashikule, and Datun regions. The used available databases for possible impact factors, respectively, are stratum and lithology, structure characteristics, volcanic activities, water chemical characteristics, geothermal gradient, seismic activities, and hot water temperature.

2.2. The Mean Variance Method. Mean variance method is a method for solving decision weight coefficient with multiindex $[24,25]$, where the weight for each impact factor can depend on the relative dispersion degree of sample database. In general, as an uncertainty open system, geothermal occurrences are controlled by the various factors and vary significantly across different regions and periods. Thus, it is difficult to accurately express the geothermal distribution only according to the traditional objective weighting method due to the uncertainty and deficient database [26, 27]. Thus, with the help of the mean variance theory, a progress description for conversion of objective weights was put forward as follows, where the weight expressed a favorability degree of the uncertainty geothermal assessment appropriately:

First, set a standardized evaluation matrix as follows:

$$
R=\left[\begin{array}{cccc}
r_{1,1} & r_{1,2} & \cdots & r_{1, j} \\
r_{2,1} & r_{2,2} & \cdots & r_{2, j} \\
\cdots & \cdots & \cdots & \cdots \\
r_{i, 1} & r_{i, 2} & \cdots & r_{i, j}
\end{array}\right]
$$

where $r_{i, j}$ is standardized index for the $j$ th class of the $i$ th impact factors and $R$ is standardized evaluation matrix.

Second, calculate the average standardized index with the equation as follows:

$$
E_{i}=\frac{1}{n} \sum_{j=1}^{n} r_{i j},
$$

where $E_{i}$ represents the average standardized index for $i$ th impact factors. 
Third, calculate the mean variance values for each impact factor; the formula was proposed as follows:

$$
F_{i}=\sqrt{\sum_{j=1}^{n}\left(r_{i j}-E_{i}\right)^{2}},
$$

where $F_{i}$ is the mean variance index for $i$ th impact factor. Hence, an objective weight value $w_{i}$ for various influencing factors is determined and the equation was put forward as follows:

$$
w_{i}=\frac{F_{i}}{\sum_{i=1}^{n} F_{i}},
$$

where $w_{i}$ is the objective weight value for $i$ th impact factor.

2.3. Coupling Empowerment Rule. The abovementioned judgment matrix in AHP and the mean variance method were considered as subjective method and objective method, according to the subjective experience and entirely the law of objective data, respectively. Thus a coupling empowerment rule was proposed by coupling AHP with mean variance method, in order to reach an agreement on the intuitive understanding for the potential geothermal phenomena in the field and the law of objective survey data [28].

To generate coupling weights for impact factors, a detailed procedure was proposed as follows:

First, set a distance function with two parameters as follows:

$$
d\left(a_{i}, b_{i}\right)=\left[\frac{1}{2} \sum_{i=1}^{n}\left(a_{i}-b_{i}\right)\right]^{1 / 2},
$$

where $a_{i}$ is the obtained weight for $i$ th impact factor by the analytic hierarchy process, $b_{i}$ is the calculated weight for $i$ th impact factor by the mean variance method, and $d\left(a_{i}, b_{i}\right)$ is deemed to be distance value $i$ th impact factor between weights $a_{i}$ and $b_{i}$.

Second, set the coupling weights as follows:

$$
w_{z}=A a_{i}+B b_{i}
$$

where $w_{z}$ is the coupling weight for $i$ th impact factor and combines a linear relationship between AHP and the mean variance method; $A$ or $B$ is the coupling coefficient of the two kinds of weights.

Third, in order to reach an agreement on the subjective process and objective method, (5) was reset as follows to obtain the corresponding coupling coefficients:

$$
d\left(a_{i}, b_{i}\right)^{2}=(A-B)^{2}, \quad A+B=1,
$$

where " $A+B=1$ " means the constraint condition of the coupling coefficient. The coupling coefficients $A$ and $B$ were calculated by combining (5) with (7), and then the integrated weight $w_{z}$ for $i$ th impact factor was obtained by substituting (6) with the its corresponding coefficients $A$ and $B$. The coupling weight method can make full use of the attribute optimization ability of analytic hierarchy process

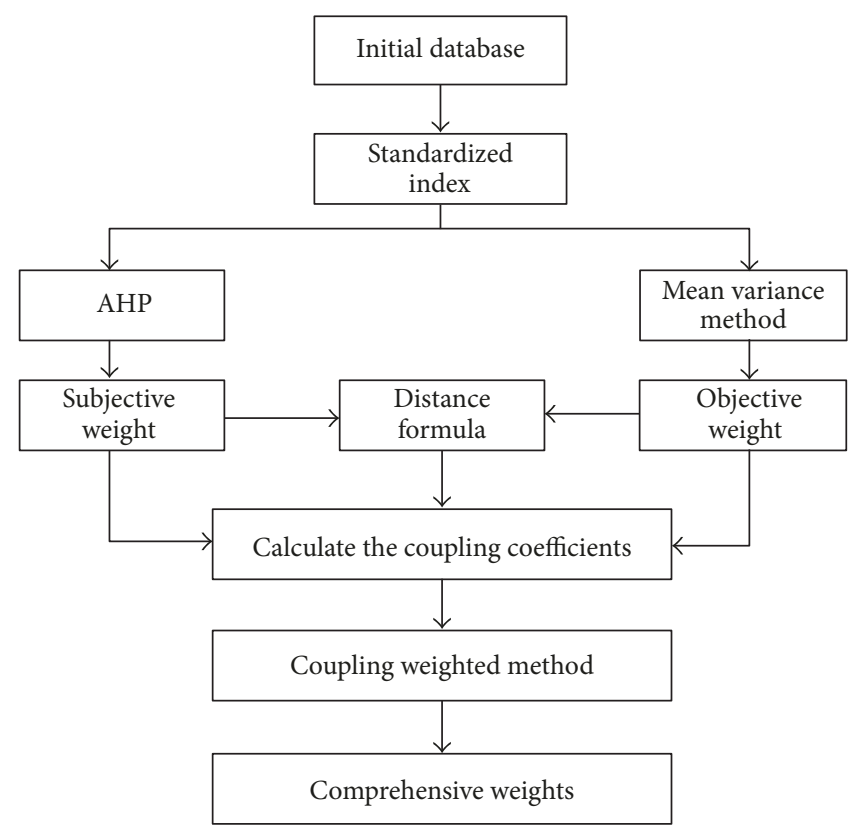

Figure 2: Schematic diagram for calculating the coupling weights.

and the objective ability of data mining of the mean variance method. Hence by using the method, the interference of human factors can be removed effectively and the singular data in data processing can be reasonably eliminated. The abovementioned calculation process of the coupling weights was performed in a schematic diagram Figure 2.

2.4. Extension Theory. As a cross discipline of originality, the extension theory was first proposed by Wen [29], and the quantitative tool has been used to study the transformation process that included system's characteristics, connotation, denotation, feasibility, and logicality. Hence, a matterelement was considered as the logical cell of extension theory and described as a formula $U=\left(N_{i}, P, V\right)$, where the three fundamental elements of the matter-element $N, P$, and $V$, respectively, were the names, characteristics, and attribute values. Matter-element can reflect the relationship between the quality and quantity with the equation $V=N(P)$. Thus, a real axis was constructed to reflect this correlation properly. The domain $\rho\left(x_{0}, X_{0}\right)$ between the point $x_{0}$ and interval $X_{0}=$ $(a, b)$ on the real axis was expressed as follows:

$$
\rho\left(x_{0}, X_{0}\right)=\left|x_{0}-\frac{a+b}{2}\right|-\frac{1}{2}(b-a) \text {. }
$$

The relationship between the distance $d\left(x_{0}, X_{0}\right)$ and domain $\rho\left(x_{0}, X_{0}\right)$ was proposed as follows, with the point $x_{0}$ and the interval $X_{0}=(a, b)$ :

$$
\begin{aligned}
& \rho=d \geq 0, \quad x \notin X_{0}, \\
& \rho<0, \\
& d=0,
\end{aligned}
$$




$$
\begin{array}{r}
x \in X_{0}, \quad x_{0} \neq a, b \\
\rho\left(x_{1}, X_{0}\right) \neq \rho\left(x_{2}, X_{0}\right), \quad x_{1}, x_{2} \in X_{0} .
\end{array}
$$

Correlation function is based on distance. It expands the qualitative description with "certain nature $P$ " to a quantitative description with "the degree of the nature $P$." The elementary dependent function was established which can make the correlation function be described with the formula as below:

$$
k(x)=\frac{\rho\left(x, X_{0}\right)}{\rho(x, X)-\rho\left(x, X_{0}\right)} .
$$

The procedure of the extenics classification algorithm for geothermal potential assessment was proposed as follows:

First, define the matter-element for the geothermal potential assessment as follows:

$$
U_{o i}=\left(N_{i}, P, V\right)=\left[\begin{array}{ccc}
N_{i} & P_{1} & V_{i 1} \\
& P_{2} & V_{i 2} \\
& \vdots & \vdots \\
& P_{n} & V_{i n}
\end{array}\right]
$$

where $N_{i}$ is the $i$ th rank of evaluated geothermal potential regions, $P_{n}$ is the $n$th character within the $i$ th rank, and $V_{i n}$ is the attribute value for $n$th character of $i$ th rank.

Second, define classical domain and the limited domain as follows:

$$
\begin{aligned}
U_{i} & =\left(N_{i}, P, V\right)=\left[\begin{array}{ccc}
N_{i} & P_{1} & V_{i 1} \\
& P_{2} & V_{i 2} \\
& \vdots & \vdots \\
& P_{n} & V_{i n}
\end{array}\right] \\
& =\left[\begin{array}{ccc}
N_{i} & P_{1} & \left\langle a_{i 1}, b_{i 1}\right\rangle \\
& P_{2} & \left\langle a_{i 2}, b_{i 2}\right\rangle \\
& \vdots & \\
& & \\
& P_{n} & \left\langle a_{i n}, b_{i n}\right\rangle
\end{array}\right],
\end{aligned}
$$

where $N_{i}$ is the susceptibility degree. The characters here referred just to the evaluation indexes. $V_{\text {in }}$ is a value scale $i$ th degree of $n$th character, which is the classical domain for normalized evaluation index. Similarly, the limited domain was proposed as follows:

$$
U_{p}=\left(P, C, Y_{p}\right)=\left[\begin{array}{ccc}
P & C_{1} & Y_{p 1} \\
& C_{2} & Y_{P 2} \\
\vdots & \vdots \\
& C_{n} & Y_{p n}
\end{array}\right]
$$

$$
=\left[\begin{array}{ccc}
P & C_{1} & \left\langle a_{p 1}, b_{p 1}\right\rangle \\
& C_{2} & \left\langle a_{p 2}, b_{p 2}\right\rangle \\
\vdots & \vdots \\
& C_{n} & \left\langle a_{p n}, b_{p n}\right\rangle
\end{array}\right],
$$

where $P$ denotes the overall partition of suitability grades and $Y_{p n}$ is the limited domain with respect to character $C_{j}$.

Third, define the matter-element to be evaluated.

The evaluation information was collected by the kind of geothermal energy level and a matter-element was proposed as follows:

$$
U_{i o}=\left(N_{i o}, C, X_{i}\right)=\left[\begin{array}{ccc}
N_{i o} & C_{1} & X_{i 1} \\
& C_{2} & X_{i 2} \\
& \vdots & \vdots \\
& C_{n} & X_{m}
\end{array}\right]
$$

where $N_{i o}$ is the matter-element for $i$ th impact factor, $C_{j}$ is $i$ th the impact factors, $X_{i j}$ is the $j$ th interval value of $i$ th impact factor, the values are corresponding to the magnitudes, and $C_{j}$ is the specific data collected from the geothermal evaluation.

Fourth, calculate the correlation degree of evaluation values for geothermal potential assessment.

The correlation $k_{i j}\left(x_{i j}\right)$ is the $j$ th factor of the $i$ th geothermal potential regions with respect to the geothermal category:

$$
k_{i j}\left(x_{i j}\right)= \begin{cases}\frac{-\rho\left(x_{i j}, X_{o i j}\right)}{\left|X_{o i j}\right|} & x_{i j} \in X_{o i j} \\ \frac{\rho\left(x_{i j}, X_{o i j}\right)}{\rho\left(x_{i j}, X_{p j}\right)-\rho\left(x_{i j}, X_{o i j}\right)} & x_{i j} \notin X_{o i j},\end{cases}
$$

where

$$
\begin{aligned}
& \rho\left(x_{i j}, X_{o i j}\right)=\left|x_{i j}-\frac{1}{2}\left(a_{o i j}+b_{o i j}\right)\right|-\frac{1}{2}\left(b_{o i j}-a_{o i j}\right), \\
& \rho\left(x_{i j}, X_{p j}\right)=\left|x_{i j}-\frac{1}{2}\left(a_{p j}+b_{p j}\right)\right|-\frac{1}{2}\left(b_{p j}-a_{p j}\right) .
\end{aligned}
$$

Fifth, calculate the correlation degree of the evaluation rank in geothermal evaluation.

The correlation of the geothermal potential $k_{i j}\left(N_{i}\right)$ related to evaluation level is

$$
k_{i j}\left(N_{i}\right)=\sum W_{i j} k_{i j}\left(x_{i j}\right)
$$

where $W_{i j}$ is the weighting distribution coefficient of the evaluation factor, and it was calculated by the formula below:

$$
W_{i j}=\frac{\left(x_{i j} / b_{o i j}\right)}{\sum_{j=1}^{n} x / b_{o i j}},
$$


TABLE 1: Impact factor indicators and variation range.

\begin{tabular}{|c|c|c|c|c|c|c|c|c|}
\hline$R$ & Name of volcanic field & $S_{1}$ & $S_{2}$ & $S_{3}$ & $S_{4}$ & $S_{5}$ & $S_{6}$ & $S_{7}$ \\
\hline 1 & Wudalianchi & 3 & 3.5 & 4 & 3 & 2 & 0.9 & 60 \\
\hline 2 & Jingpohu & 3 & 2.5 & 3 & 2 & 3 & 0.9 & 60 \\
\hline 3 & Longgang & 3 & 2.5 & 4 & 1.5 & 3 & 0.5 & 68 \\
\hline 4 & Changbaishan & 3 & 3.5 & 4 & 2.5 & 3 & 0.5 & 82 \\
\hline 5 & Aershan & 4 & 3 & 3.5 & 2.5 & 2 & 0.7 & 48 \\
\hline 6 & Wulanhada & 3 & 2.5 & 3 & 2 & 2 & 0.9 & 60 \\
\hline 7 & Datong & 3 & 3 & 2 & 2 & 3 & 0.9 & 63 \\
\hline 8 & Qiongbei & 3 & 3 & 4 & 2 & 3 & 1 & 80 \\
\hline 9 & Kekexili & 2 & 2.5 & 2 & 1.5 & 3 & 1 & 70 \\
\hline 10 & Ashikule & 2 & 2 & 3.5 & 1.5 & 2 & 1 & 40 \\
\hline 11 & Datun & 3 & 3 & 3.5 & 3.5 & 4 & 1 & 10 \\
\hline Index parameters & Range & $1 \sim 4$ & $1 \sim 4$ & $1 \sim 4$ & $1 \sim 4$ & $1 \sim 4$ & $0 \sim 1$ & $0 \sim 100$ \\
\hline
\end{tabular}

apparently,

$$
\sum_{j=1}^{n} W_{i j}=1 .
$$

Sixth, determine geothermal potential rank.

After calculating the correlation $k_{i j}\left(N_{i}\right)$ for impact factor $N_{i}$ related to evaluation level with the formula $k_{i t}\left(N_{i}\right)=$ $\max \left\{k_{i t o}\left(N_{i}\right)\right\}$, the potential evaluation level for categories was obtained qualitatively. The value and the relationship of $k_{i t}\left(N_{i}\right)$ can reflect the potential of the $i$ th impact factor and the degree of category.

\section{Application with Coupling Weight-Based Extenics Method}

3.1. Database Preparation. Plenty of hydrothermal activities in China including volcanos, hot springs, and hot water wells have been well studied, and the basic geological and heat flow databases for geothermal emergencies regions were also accurately collected and sorted out $[30,31]$. In this paper, there are 11 famous volcanic areas and seven specific geological conditions were selected to evaluate the geothermal potential regions. The selected impact factors, respectively, were stratum and lithology named $S_{1}$, structure characteristics $\left(S_{2}\right)$, volcanic activities $\left(S_{3}\right)$, water chemical characteristics $\left(S_{4}\right)$, geothermal gradient $\left({ }^{\circ} \mathrm{C} / 100 \mathrm{~m} ; S_{5}\right)$, earthquake activities $\left(S_{6}\right)$, and hot spring temperature $\left({ }^{\circ} \mathrm{C}\right.$; $\left.S_{7}\right)$. The index of each impact factor was shown in Table 1.

The selected impact factors were put forward as follows:

Stratum and Lithology $\left(S_{1}\right)$. A stratum is the place to store the geothermal energy where the thermal storage and conductivity vary significantly in different lithologies, and only the good stratum and lithology can store rich geothermal resources.
Structure Characteristics $\left(S_{2}\right)$. Faults have been well developed in areas with plenty of active structures, which provided channels for the underground hot water migration. Thus, the active structure is closely related to geothermal energy.

Volcanic Activities $\left(S_{3}\right)$. Magmatic activities in the volcano regions can provide the heat source for the geothermal field; hence they are directly related to the existence of geothermal energy.

Water Chemical Characteristics $\left(S_{4}\right)$. Hydrochemical characteristics of the geothermal fluid (ionic composition and mineralization degree) have inner correlations with the geological tectonic activities and the corresponding thermal reservoir.

Geothermal Gradient $\left(S_{5}\right)$. Geothermal gradients were composed of lines contracted from the higher temperature; thus a higher temperature for land surface can indicate the more abundant geothermal resources.

Earthquake Activities $\left(S_{6}\right)$. Seismic activities are closely related to the active structure; hence a large seismic level in history usually indicates the development of the fault structure and volcanic activities.

Hot Spring Temperature $\left(S_{7}\right)$. The land surface temperature anomalies are deemed to be a reference indicator for the geothermal emergencies, where higher temperature means more possibility of geothermal occurrences.

All the selected impact factors were determined by qualitative methods, and the influence of each factor was divided into ranges. According to a previous experience and a large number of statistical data [31], the variation range of influencing factors is shown in Table 1.

3.2. Coupling Weight Calculation. According to the statistics and analysis of a large number of data, the selected impact factors were divided into 3 grades by single factor method and the results were presented in Table 2. 
TABLE 2: Dimensionless quantization and standardized index level for impact factors.

\begin{tabular}{|c|c|c|c|c|c|c|}
\hline \multirow{2}{*}{ Impact factors } & \multicolumn{3}{|c|}{ Index level } & \multicolumn{3}{|c|}{ Standardized index level } \\
\hline & High & Medium & Low & High & Medium & Low \\
\hline Formation and lithology & $3 \sim 4$ & $2 \sim 3$ & $1 \sim 2$ & $0.67 \sim 1.00$ & $0.33 \sim 0.60$ & $0.00 \sim 0.33$ \\
\hline Structure characteristics & $3.6 \sim 4.1$ & $2.6 \sim 3.6$ & $1.0 \sim 2.6$ & $0.84 \sim 1.00$ & $0.52 \sim 0.84$ & $0.00 \sim 0.55$ \\
\hline Volcanic activity & $3.6 \sim 4.1$ & $2.6 \sim 3.6$ & $1.0 \sim 2.6$ & $0.84 \sim 1.00$ & $0.52 \sim 0.84$ & $0.00 \sim 0.52$ \\
\hline $\begin{array}{l}\text { Water chemical } \\
\text { characteristics }\end{array}$ & $3.6 \sim 4.1$ & $2.6 \sim 3.6$ & $1.0 \sim 2.6$ & $0.84 \sim 1.00$ & $0.52 \sim 0.84$ & $0.00 \sim 0.52$ \\
\hline Geothermal gradient & $3 \sim 4$ & $2 \sim 3$ & $1 \sim 2$ & $0.84 \sim 1.00$ & $0.52 \sim 0.84$ & $0.00 \sim 0.52$ \\
\hline Earthquake activity & $0.8 \sim 1.0$ & $0.4 \sim 0.8$ & $0.0 \sim 0.4$ & $0.80 \sim 1.00$ & $0.40 \sim 0.80$ & $0.00 \sim 0.40$ \\
\hline Hot spring temperature & $80 \sim 100$ & $50 \sim 80$ & $0.0 \sim 50$ & $0.80 \sim 1.00$ & $0.00 \sim 0.40$ & $0.00 \sim 0.50$ \\
\hline
\end{tabular}

TABLE 3: Standardized attribute values for geothermal potential regions.

\begin{tabular}{lccccccccc}
\hline$n$ & $\begin{array}{c}\text { Geothermal potential } \\
\text { regions }\end{array}$ & $S_{0}$ & $S_{1}$ & $S_{2}$ & $S_{3}$ & $S_{4}$ & $S_{5}$ & $S_{6}$ & $S_{7}$ \\
\hline 1 & Wudalianchi & High & 0.67 & 0.81 & 0.97 & 0.64 & 0.32 & 0.90 & 0.60 \\
2 & Jingpohu & Medium & 0.83 & 0.48 & 0.65 & 0.32 & 0.65 & 0.90 & 0.60 \\
3 & Longgang & High & 0.83 & 0.48 & 0.97 & 0.81 & 0.65 & 0.50 & 0.81 \\
4 & Changbaishan & High & 0.83 & 0.81 & 0.97 & 0.48 & 0.65 & 0.50 & 0.82 \\
5 & Aershan & Medium & 1.00 & 0.65 & 0.81 & 0.48 & 0.32 & 0.70 & 0.48 \\
6 & Wulanhada & Medium & 0.50 & 0.65 & 0.32 & 0.16 & 0.32 & 0.50 & 0.83 \\
7 & Datong & Medium & 0.83 & 0.65 & 0.32 & 0.32 & 0.65 & 0.90 & 0.63 \\
8 & Qiongbei & Medium & 0.83 & 0.65 & 0.97 & 0.32 & 0.65 & 1.00 & 0.76 \\
9 & Kekexili & Low & 0.33 & 0.48 & 0.48 & 0.61 & 0.65 & 1.00 \\
10 & Ashikule & Low & 0.33 & 0.32 & 0.81 & 0.61 & 0.32 & 1.00 \\
11 & Datun & Medium & 0.67 & 0.65 & 0.81 & 0.81 & 0.97 & 1.00 \\
\hline
\end{tabular}

TABLE 4: The coupling weights for all used impact factors.

\begin{tabular}{lcccccc}
\hline $\begin{array}{l}\text { Formation and } \\
\text { lithology }\end{array}$ & $\begin{array}{c}\text { Structure } \\
\text { characteristics }\end{array}$ & $\begin{array}{c}\text { Volcanic } \\
\text { activity }\end{array}$ & $\begin{array}{c}\text { Water chemical } \\
\text { characteristics }\end{array}$ & $\begin{array}{c}\text { Geothermal } \\
\text { gradient }\end{array}$ & $\begin{array}{c}\text { Earthquake } \\
\text { activity }\end{array}$ & $\begin{array}{c}\text { Hot spring } \\
\text { temperature }\end{array}$ \\
\hline 0.1014 & 0.2001 & 0.1215 & 0.0773 & 0.3151 & 0.0504 & 0.1342 \\
\hline
\end{tabular}

In order to calculate conveniently, it is necessary to standardize the index factors mentioned in Table 1 and the equation was proposed as follows:

$$
n_{i j}^{\prime}=\frac{\left(n_{i j}-n_{i j}^{\min }\right)}{\left(n_{i j}^{\max }-n_{i j}^{\min }\right)},
$$

where $n_{i j}$ is the attribute value for $j$ th impact factor of $i$ th selected geothermal regions, $n_{i j}{ }^{\max }$ is the maximum attribute value, $n_{i j}$ min is the minimum attribute value of $j$ factor, and $n^{\prime}{ }_{i j}$ is the standardized value for $j$ th impact factor of $i$ th study areas. The calculated results were put forward as shown in Table 3.

The abovementioned parameter $S_{0}$ for geothermal potential regions was determined with a reference in Table 2, and then the coupling weights were obtained according to process of Figure 2. The results of comprehensive weights were presented as shown in Table 4.

3.3. Matter-Element Assessment. According to the standardized data in Table 3, the classical domain matrix for matterelement and joint domain matter-element were structured as follows:

$$
R_{01}=\left[\begin{array}{ccc}
N_{01} & C_{1} & \langle 0.67,1.0\rangle \\
& C_{2} & \langle 0.84,1.0\rangle \\
& C_{3} & \langle 0.84,1.0\rangle \\
& C_{4} & \langle 0.84,1.0\rangle \\
& C_{5} & \langle 0.67,1.0\rangle \\
& C_{6} & \langle 0.8,1.0\rangle \\
& C_{7} & \langle 0.8,1.0\rangle
\end{array}\right],
$$


TABLE 5: Comparison of calculated and actual results in Wudalianchi.

\begin{tabular}{lccccc}
\hline$N$ & Low & Medium & High & Evaluation grade & Actual results \\
\hline 1 & -0.3543 & -0.0480 & -0.0209 & High & High \\
2 & -0.2684 & 0.3283 & -0.1617 & Medium & Medium \\
3 & -0.5912 & -0.2079 & 0.0623 & High & High \\
4 & -0.5630 & -0.0454 & 0.1010 & High & High \\
5 & -0.2834 & -0.0097 & -0.2118 & Medium & Medium \\
6 & 0.2236 & 0.0242 & -0.4628 & Medium & Medium \\
7 & -0.1037 & 0.1955 & -0.1974 & Medium & Medium \\
8 & -0.4962 & 0.0481 & -0.0127 & Low & Low \\
9 & 0.1775 & -0.0096 & -0.4574 & Low & Low \\
10 & 0.2314 & -0.2424 & -0.4710 & -0.0883 & Medium \\
11 & -0.6071 & 0.1145 & & & Medium \\
\hline
\end{tabular}

$$
\begin{aligned}
& R_{02}=\left[\begin{array}{ccc}
N_{02} & C_{1} & \langle 0.33,0.67\rangle \\
& C_{2} & \langle 0.52,0.84\rangle \\
& C_{3} & \langle 0.52,0.84\rangle \\
& C_{4} & \langle 0.52,0.84\rangle \\
& C_{5} & \langle 0.33,0.67\rangle \\
& C_{6} & \langle 0.4,0.8\rangle \\
& C_{7} & \langle 0.5,0.8\rangle
\end{array}\right], \\
& R_{03}=\left[\begin{array}{ccc}
N_{03} & C_{1} & \langle 0,0.33\rangle \\
& C_{2} & \langle 0,0.52\rangle \\
& C_{3} & \langle 0,0.52\rangle \\
& C_{4} & \langle 0,0.52\rangle \\
& C_{5} & \langle 0,0.33\rangle \\
& C_{6} & \langle 0,0.4\rangle \\
& C_{7} & \langle 0,0.5\rangle
\end{array}\right],
\end{aligned}
$$$$
R_{p}=\left[\begin{array}{ccc}
P & C_{1} & \langle 0,1.0\rangle \\
& C_{2} & \langle 0,1.0\rangle \\
& C_{3} & \langle 0,1.0\rangle \\
& C_{4} & \langle 0,1.0\rangle \\
& C_{5} & \langle 0,1.0\rangle \\
& C_{6} & \langle 0,1.0\rangle \\
& C_{7} & \langle 0,1.0\rangle
\end{array}\right],
$$$$
R_{1}=\left[\begin{array}{ccc}
N_{01} & C_{1} & 0.67 \\
& C_{2} & 0.81 \\
& C_{3} & 0.97 \\
& C_{4} & 0.65 \\
& C_{5} & 1.00 \\
& C_{6} & 0.90 \\
& C_{7} & 0.60
\end{array}\right],
$$

\section{Conclusions}

In this paper, to overcome the subjective and objective disadvantages in weighting calculation, an extension theorybased method was proposed by coupling the process of AHP and mean variance method, in order to reach an agreement on the intuitive understanding for the selected geothermal impact factors and their objective database. The improved coupling weights were used in classification of geothermal potential assessment, by replacing the point values with intervals according to extension judgment matrix. The evaluation results reflected the actual situation well and can play an indicative role in the partition of the geological 
conditions that may have inner connections with geothermal emergencies. An example put forward in Wudalianchi region was used in analysis and verified, and the results showed perfect performance where 10 out of 11 impact factors agree with the actual evaluation grades. Therefore, the study can objectively provide a guidance for initial phase of geothermal investigation. However, the application of improved classification method in geothermal potential evaluation, especially in the aspect of parameter selection, should be given further improvement because the selected impact factors may not be considered completely.

\section{Conflicts of Interest}

The authors declare that there are no conflicts of interest regarding the publication of this paper.

\section{References}

[1] State Bureau of Technical Supervision, "Standards of geothermal resources geological investigation (GB116-89)," Tech. Rep., 1990.

[2] W. Jiyang and S. Zhanxue, Magic Geothermal, Tsinghua University press, Beijing, China, 2001.

[3] D. E. White, "Assessment of geothermal resources of the United States," in Circular, D. L. Williams, Ed., vol. 726, 1975.

[4] Z. Yunzhang, C. Ruhan, and Z. Bida, "A discussion on geothermal resources and their development," Acta Geological Sichuan, vol. 1, no. 21, pp. 23-24, 2001.

[5] "Evaluating method of geothermal resource (Dz40-85)," Tech. Rep., Standards press of China, Beijing, China, 1985.

[6] W. Guiling, Z. Fawang, and L. Zhiming, "An analysis of present situation and prospects of geothermal energy development and utilization in the World," Acta Geoscientica Sinica, vol. 21, no. 2, 2000.

[7] W. Jiyang, L. Shibin, and Z. Huazhou, "Development strategy of China geothermal resource energy in 21st century," Electric Power of China, vol. 33, no. 09, 2000.

[8] Lin, Z. Xiuhua, and Z. Meiping, "The utilization and development prospect of geothermal energy resource," Resources \& Industries, vol. 3, no. 8, pp. 20-23, 2006.

[9] ENEL, "Geothermal Power development studies in Iran, General Report to the Ministry of Energy," Tech. Rep., Islamic Republic of Iran, 1983.

[10] D. Lu, "Fuzzy Comprehensive Evaluation on Sustainable Exploitation and Utilization Potential of Karst Geothermal Resources," Site Investigation Science \& Technology, vol. 406, no. 3, pp. 393-410, 2008.

[11] J. Jokinen and I. T. Kukkonen, "Inverse simulation of the lithospheric thermal regime using the Monte Carlo method," Tectonophysics, vol. 306, no. 3-4, pp. 293-310, 1999.

[12] J. Zhang, J. W. Lin, L. Lin et al., "Evaluation of deep geothermal water sustainable development ability based on AHP and BP neural network," Ground Water, vol. 43, no. 3, pp. 223-229, 2008.

[13] K. K. Abdul Azeez and T. Harinarayana, "Magnetotelluric evidence of potential geothermal resource in Puga, Ladakh, NW Himalaya," Current Science, vol. 93, no. 3, pp. 323-329, 2007.

[14] G. L. Raines and M. J. Mihalasky, "A reconnaissance method for delineation of tracts for regional-scale mineral-resource assessment based on geologic-map data," Natural Resources Research, vol. 11, no. 4, pp. 241-248, 2002.
[15] M. F. Coolbaugh and L. A. Shevenell, "A method for estimating undiscovered geothermal resources in Nevada and the Great Basin," in Proceedings of the Geothermal Energy: The Reliable Renewable - Geothermal Resources Council 2004 Annual Meeting, GRC, pp. 13-18, September 2004.

[16] M. T. Hussein, A. Lashin, A. Al Bassam, N. Al Arifi, and I. Al Zahrani, "Geothermal power potential at the western coastal part of Saudi Arabia," Renewable \& Sustainable Energy Reviews, vol. 26, pp. 668-684, 2013.

[17] V. Paoletti, G. Langella, R. Di Napoli et al., "A tool for evaluating geothermal power exploitability and its application to Ischia, Southern Italy," Applied Energy, vol. 139, pp. 303-312, 2015.

[18] K. Nagasaka and M. Rumbayan, "Development of priority decision for renewable energy potential using analytical hierarchy process and geographical information system method," International Journal of Advanced Mechatronic Systems, vol. 5, no. 4, pp. 270-278, 2013.

[19] B. Sadeghi and M. Khalajmasoumi, "A futuristic review for evaluation of geothermal potentials using fuzzy logic and binary index overlay in GIS environment," Renewable \& Sustainable Energy Reviews, vol. 43, pp. 818-831, 2014.

[20] C. Augustine, "Analysis of sedimentary geothermal systems using an analytical reservoir model," in Proceedings of the Geothermal Resources Council Annual Meeting - Geothermal: A Global Solution, GRC 2014, pp. 641-647, October 2014.

[21] T. L. Saaty, "Applications of analytical hierarchies," Mathematics and Computers in Simulation, vol. 21, no. 1, pp. 1-20, 1979.

[22] C. Wei, C. Chien, and M. J. Wang, "An AHP-based approach to ERP system selection," International Journal of Production Economics, vol. 96, no. 1, pp. 47-62, 2005.

[23] J. G. Dolan, "Shared decision-making-transferring research into practice: The Analytic Hierarchy Process (AHP)," Patient Education and Counseling, vol. 11, no. 1, pp. 21-30, 2008.

[24] D. J. Brus and N. P. A. Saby, "Approximating the variance of estimated means for systematic random sampling, illustrated with data of the French Soil Monitoring Network," Geoderma, vol. 279, pp. 77-86, 2016.

[25] P. Ruan, J. Shen, R. M. Santella, S. Zhou, and S. Wang, "NEpiC: A network-assisted algorithm for epigenetic studies using mean and variance combined signals," Nucleic Acids Research, vol. 44, no. 16, article no. e134, 2016.

[26] N. Balakrishnan and P. S. Chan, "Order statistics from extreme value distribution i: Tables of means, variances and covariances," Communications in Statistics - Simulation and Computation, vol. 21, no. 4, pp. 1199-1217, 1992.

[27] R. I. Cukier, "Variance of a potential of mean force obtained using the weighted histogram analysis method," The Journal of Physical Chemistry B, vol. 117, no. 47, pp. 14785-14796, 2013.

[28] R. S. Parrish, "Computing variances and covariances of normal order statistics," Communications in Statistics - Simulation and Computation, vol. 21, no. 1, pp. 71-101, 1992.

[29] C. Wen, "Introduction of extenics," Systems Engineering-Theory \& Practice, vol. 1, pp. 76-84, 1998.

[30] L. Canren and W. Baotai, "Development and utilization of geothermal," Yearbook of Nature Science, pp. 37-47, 1987.

[31] L. Lujuan, The Asian Geothermal Map And The Evaluation on Geothermal Potential, Jilin University, Changchun, China, 2011. 


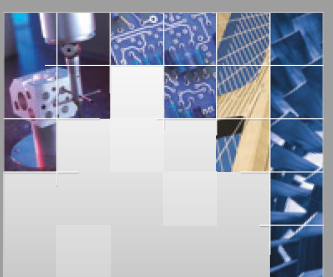

\section{Enfincering}
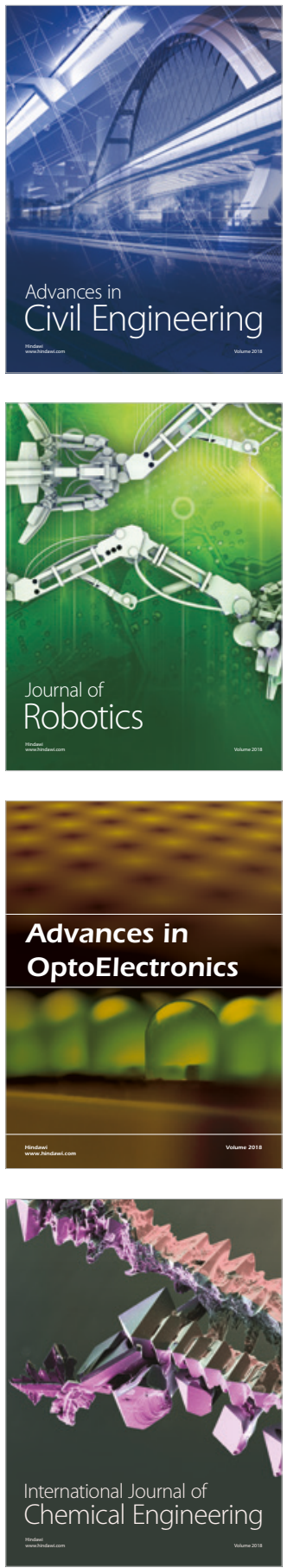

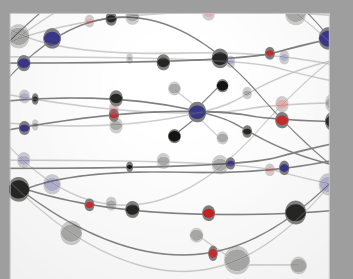

\section{Rotating \\ Machinery}

The Scientific World Journal

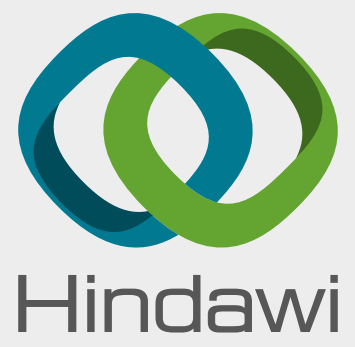

Submit your manuscripts at

www.hindawi.com
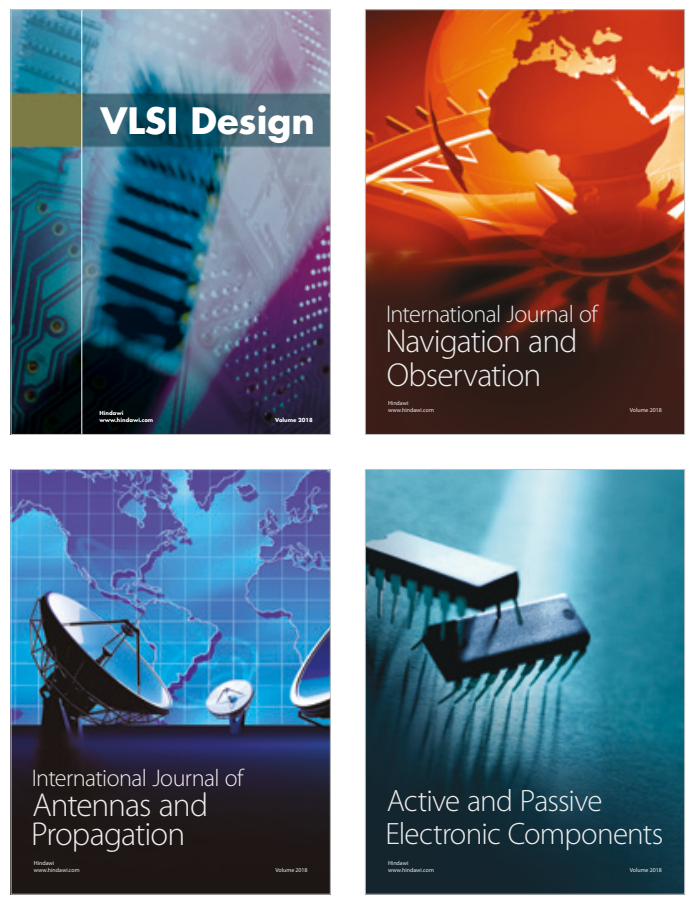
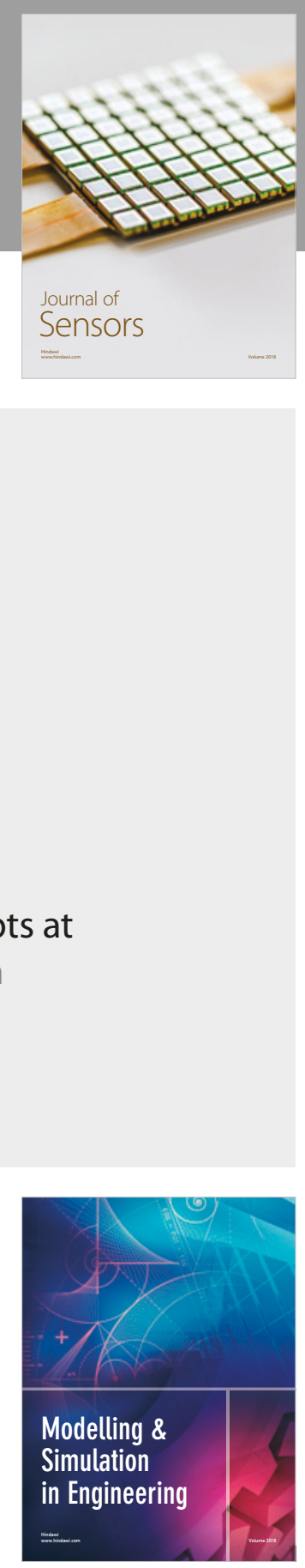

\section{Advances \\ Multimedia}
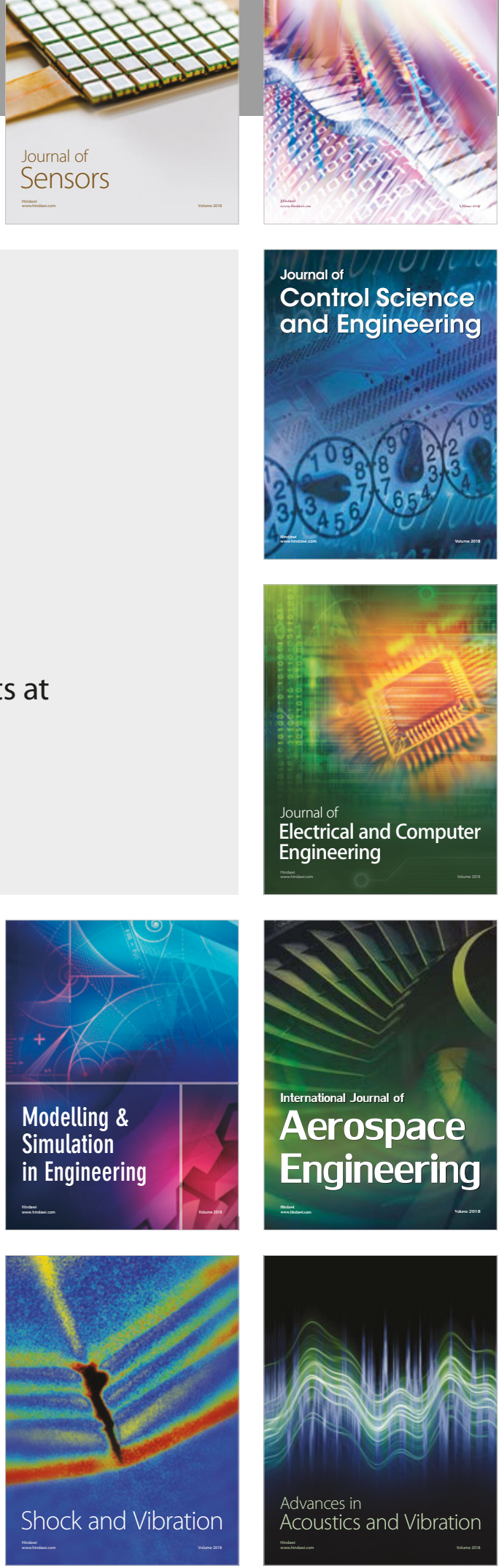